DFPD-00/TH/40

\title{
Constraints on R-parity violating stop couplings from flavor physics
}

\author{
Pietro Slavich円 \\ Dipartimento di Fisica, Università di Padova and \\ INFN, Sezione di Padova, I-35131 Padova, Italy
}

\begin{abstract}
We perform a critical reassessment of the constraints on the R-parity and baryon number violating (s)top couplings coming from flavor physics. In particular, we study $K^{0}-\bar{K}^{0}$ mixing, including QCD corrections and a class of diagrams that were neglected in previous analyses: both effects give sizeable contributions. We explain how the resulting bounds can be translated into constraints on individual couplings in the simplest class of flavor models, based on $\mathrm{U}(1)$ horizontal symmetries. We finally discuss the implications for the possibility of observing single superparticle production at the Tevatron.
\end{abstract}

\footnotetext{
${ }^{1}$ e-mail address: slavich@pd.infn.it
} 


\section{Introduction}

In the Minimal Supersymmetric Standard Model (MSSM) it is assumed that R-parity $\left(R_{p}\right)$ is conserved [1]. While the particles of the Standard Model are even under $R_{p}$, their supersymmetric partners are odd, thus the latter can only be produced in pairs and they always decay into final states involving an odd number of supersymmetric particles. Although it considerably simplifies the structure of the MSSM, the conservation of Rparity has no firm theoretical justification (for a review and references on versions of the MSSM with broken R-parity, see e.g. [2]). The most general $R_{p}$-violating superpotential that can be written with the MSSM superfields contains both lepton number and baryon number violating terms. Their simultaneous presence is in fact strongly limited, since it would induce fast proton decay. However, it is possible to allow for the presence of baryon number violating couplings only [3], coming from the superpotential

$$
\mathcal{W}_{B \beta}=\lambda_{i j k}^{\prime \prime} \epsilon^{\alpha \beta \gamma} U_{i \alpha}^{c} D_{j \beta}^{c} D_{k \gamma}^{c},
$$

where $U^{c}$ and $D^{c}$ (c denotes charge conjugation) are the chiral superfields associated with $\mathrm{SU}(2)$-singlet antiquarks, in a basis where the quark masses are diagonal, $i, j, k=1,2,3$ are flavor indices and $\alpha, \beta, \gamma=1,2,3$ are color indices. The color structure of $\mathcal{W}_{\not B}$ implies that the $\lambda_{i j k}^{\prime \prime}$ are antisymmetric in the last two indices, limiting the number of independent couplings to 9 .

A number of recent works [4]-[8] studied the production of single supersymmetric particles at the Fermilab Tevatron through the R-parity and baryon number violating couplings of eq. (1) that involve a top (s)quark. These works were motivated by the consideration that, while the $R_{p}$ and baryon number violating couplings $\lambda_{1 j k}^{\prime \prime}$ which involve an up (s)quark are severely constrained by the absence of neutron-antineutron oscillations [3, 9] and double nucleon decay into kaons [10], the limits on the couplings that involve a top (s)quark are believed to be much weaker, of order $\lambda_{3 j k}^{\prime \prime}<1$ for sfermion masses heavier than $100 \mathrm{GeV}$ (see e.g. [5] and references therein). Moreover, in most scenarios of supersymmetry breaking it is reasonable to expect that the third-generation squarks are lighter than the other sfermions, due to the effect of their large Yukawa couplings on the renormalization group evolution of the masses between the ultraviolet cutoff scale of the MSSM and the weak scale. Thus, if the couplings $\lambda_{3 j k}^{\prime \prime}$ are not much smaller than 1 , and the third-generation squarks are not too heavy, it should be possible to observe single stop production in the interaction of two down-type antiquarks, or single sbottom production in the decay of a top quark.

In view of this situation, it is extremely interesting to understand how the constraints on the $R_{p}$-violating (s)top couplings that can be derived from low-energy experimental data compare with the bound $\lambda_{3 j k}^{\prime \prime}<1$ considered in Refs. [4]- [8]. In this note we will study the constraints that can be derived from flavor physics. In particular, in section 2 we will perform a careful study of the limits coming from $K^{0}-\bar{K}^{0}$ mixing, including QCD corrections and a class of diagrams that were neglected in previous analyses [10]- 
12]: both effects give sizeable contributions. We will show that $K^{0}-\bar{K}^{0}$ mixing places an upper bound of order (few) $\times 10^{-2}$ on the combination $\left|\lambda_{313}^{\prime \prime} \lambda_{323}^{\prime \prime *}\right|^{1 / 2}$ of $R_{p}$-violating (s)top couplings. For degenerate couplings, $\lambda_{312}^{\prime \prime}=\lambda_{313}^{\prime \prime}=\lambda_{323}^{\prime \prime} \equiv \lambda^{\prime \prime}$, this is significantly stronger than the bounds usually considered in the literature. In section 3 we will consider a more refined way of translating the bounds coming from $K^{0}-\bar{K}^{0}$ mixing into bounds on the individual couplings $\lambda_{3 j k}^{\prime \prime}$ : it makes use of the simplest models of flavor, those based on $\mathrm{U}(1)$ horizontal symmetries, to correlate the size of the different couplings. Finally, in section 4 we will apply our analysis to the specific scenarios considered in Refs. [4]8], and we will show that $K^{0}-\bar{K}^{0}$ mixing strongly constrains the possibility of observing single superparticle production at the Tevatron, not only under the rough assumption of degenerate couplings, but also assuming the most general hierarchies allowed by realistic $\mathrm{U}(1)$ flavor symmetries.

Before addressing our main points, we comment on some bounds that were previously discussed in the literature. The R-parity and baryon number violating interactions affect, at the one-loop level, the coupling of the $Z$ boson to the right-handed down-type quarks. The upper bounds quoted in Refs. [4]- 8 ] come from the measurement of $R_{l} \equiv \Gamma(Z \rightarrow$ hadrons $) / \Gamma\left(Z \rightarrow l^{+} l^{-}\right)$[13]. However, in order to derive a reliable bound on the couplings $\lambda_{3 j k}^{\prime \prime}$, the contributions of both $R_{p}$-violating and $R_{p}$-conserving MSSM interactions to the $Z$-peak observables must be taken into account [14. A recent analysis of the whole set of hadronic $Z$-peak observables [15] suggested that the couplings $\lambda_{3 j 3}^{\prime \prime}$ are ruled out at $95 \%$ confidence level. The reason is that, while the data ask for a positive shift in the coupling of the right-handed $b$ quarks to the $Z$ boson with respect to its Standard Model value, the $\lambda^{\prime \prime}$ corrections shift this coupling in the negative direction. However, the interpretation of these results appears to be controversial: it was shown in 15 that the bounds on $\lambda_{3 j 3}^{\prime \prime}$ coming from the $Z$-peak data are much weaker if one performs a Bayesian statistical analysis instead of the classical one.

The experimental bound on neutron-antineutron oscillations was used [9] to set limits on the $R_{p}$-violating couplings $\lambda_{1 j k}^{\prime \prime}$ which involve an up (s)quark. In a subsequent paper [16], a similar analysis was performed on a class of diagrams that also contribute to neutron-antineutron oscillations, but allow to set limits on the couplings $\lambda_{3 j k}^{\prime \prime}$. Contrary to what claimed by the authors of [16], we have found that such limits are not competitive with the ones that can be derived from flavor physics.

The authors of [11] studied the contribution of the $R_{p}$-violating terms to some rare decays of the $B^{+}$meson, namely $B^{+} \rightarrow \bar{K}^{0} K^{+}$and $B^{+} \rightarrow \bar{K}^{0} \pi^{+}$. In order to reduce the theoretical uncertainties, they considered the ratio of the partial width of the rare decay to the partial width of the decay $B^{+} \rightarrow K^{+} J / \psi$, which proceeds unsuppressed in the Standard Model. The limits on the $R_{p}$-violating couplings that can be derived in this way are less stringent than those coming from $K^{0}-\bar{K}^{0}$ mixing, but still one order of magnitude below those assumed in [4]- [8]. Using the formulae of Ref. [11], with updated values for the experimental upper bounds on the branching ratios and for the CKM matrix elements [17], 
and assuming that the lightest stop mass eigenstate is approximated by $\tilde{t}_{R}$, we obtained:

$$
\left|\lambda_{312}^{\prime \prime} \lambda_{323}^{\prime \prime *}\right|<3.0 \times 10^{-3} \frac{m_{\tilde{t}_{R}}^{2}}{m_{W}^{2}}, \quad\left|\lambda_{312}^{\prime \prime} \lambda_{313}^{\prime \prime *}\right|<3.9 \times 10^{-3} \frac{m_{\tilde{t}_{R}}^{2}}{m_{W}^{2}} .
$$

As an example we can consider the representative parameter choice recently used for the discussion of single stop production at the Tevatron (see section ther the details concerning this scenario). In the case of degenerate couplings $\lambda_{312}^{\prime \prime}=\lambda_{313}^{\prime \prime}=\lambda_{323}^{\prime \prime} \equiv \lambda^{\prime \prime}$, and taking into account the mixing in the stop sector, we obtained the upper limit $\lambda^{\prime \prime}<0.15-0.24$ for a light stop mass ranging between 180 and $325 \mathrm{GeV}$.

\section{Improved constraints from $K^{0}-\bar{K}^{0}$ mixing}

Bounds on the $R_{p}$-violating couplings can be derived from $K^{0}-\bar{K}^{0}$ mixing [10]- [12]. Flavorchanging neutral currents in SUSY models can arise in a "direct" way, when the flavor violation occurs through flavor violating vertices in the diagrams, or in an "indirect" way, due to the existence of non diagonal sfermion masses in the basis where the fermion masses are diagonal. In minimal supergravity scenarios, where the soft mass matrices at the GUT scale are flavor diagonal, non diagonal squark masses are generated by flavor violating couplings through the renormalization group equations. However, as shown in [12], their contribution to $K^{0}-\bar{K}^{0}$ mixing can be neglected. Thus, in the following we will assume that the quark and squark mass matrices are diagonalized by the same rotations.

The diagrams that give the dominant contributions to $K^{0}-\bar{K}^{0}$ mixing in minimal supergravity scenarios with R-parity violation are shown in fig. 1. The most general $\Delta S=2$ effective Lagrangian can be written as:

$$
\mathcal{L}_{\mathrm{eff}}^{\Delta S=2}=\sum_{i=1}^{5} C_{i} Q_{i}+\sum_{i=1}^{3} \tilde{C}_{i} \tilde{Q}_{i}
$$

where the four-fermion operators $Q_{i}$ and $\tilde{Q}_{i}$ are defined as in 18. The operators relevant to this analysis are $Q_{1}=\bar{d}_{L}^{\alpha} \gamma_{\mu} s_{L}^{\alpha} \bar{d}_{L}^{\beta} \gamma^{\mu} s_{L}^{\beta}$ ( $\alpha, \beta$ are color indices), coming from the Standard Model diagram (fig. 11a), $\tilde{Q}_{1}=\bar{d}_{R}^{\alpha} \gamma_{\mu} s_{R}^{\alpha} \bar{d}_{R}^{\beta} \gamma^{\mu} s_{R}^{\beta}$, coming from the diagrams with four $\lambda^{\prime \prime}$ couplings (fig. 1 $1 \mathrm{~b}-\mathrm{c}$ ), and $Q_{4}=\bar{d}_{R}^{\alpha} s_{L}^{\alpha} \bar{d}_{L}^{\beta} s_{R}^{\beta}, Q_{5}=\bar{d}_{R}^{\alpha} s_{L}^{\beta} \bar{d}_{L}^{\beta} s_{R}^{\alpha}$, coming from the diagrams with two CKM and two $\lambda^{\prime \prime}$ couplings (fig. 1 $1 \mathrm{~d}-\mathrm{e}$ ). The corresponding coefficients $C_{i}$ are evaluated at a common scale $M_{S}$, where the supersymmetric particles are integrated out. We computed all the coefficients that are relevant to the case under consideration, including the contributions of charginos and charged Higgs that were neglected in earlier analyses 10-12]: their explicit expressions are given in the Appendix.

The contribution of the effective Lagrangian $\mathcal{L}^{\Delta S=2}$ to the $K_{S}-K_{L}$ mass difference $\Delta m_{K}$ is related to the matrix element $\left\langle K^{0}\left|\mathcal{L}_{\text {eff }}^{\Delta S=2}\right| \bar{K}^{0}\right\rangle$. The coefficients $C_{i}$ must be evolved from the scale $M_{S}$, which is of order of the masses of the supersymmetric particles, down to some hadronic scale $\mu_{h}$ (e.g. $2 \mathrm{GeV}$ ) at which the matrix element can be evaluated. Moreover, 
the long-distance hadronic processes give contributions to the matrix elements $\left\langle K^{0}\left|Q_{i}\right| \bar{K}^{0}\right\rangle$ that cannot be evaluated perturbatively, and are parametrized by "bag factors" $B_{i}$ (for the explicit definitions see [18]). We have calculated the contribution of the $R_{p}$-violating couplings to $\Delta m_{K}$, using the NLO QCD evolution of the coefficients $C_{i}$ and the lattice calculations for the $B_{i}$ presented in [18]. It is interesting to note that the main effect of the QCD corrections, which were neglected in earlier analyses, is a sizeable enhancement of the coefficient $C_{4}$.

Due to the large uncertainties that affect the theoretical evaluation of $\Delta m_{K}$ in the Standard Model (see e.g. [19] and references therein), a conservative limit on the $R_{p^{-}}$ violating couplings can be derived by requiring that the contribution to $\Delta m_{K}$ of the diagrams shown in fig. $1 \mathrm{~b}$-e is not larger than the experimental value $\Delta m_{K}^{\exp }=(3.489 \pm$ $0.008) \times 10^{-15} \mathrm{GeV}$ [17]. When all the contributions of the diagrams shown in fig. [1b-e are taken into account, the resulting limit on the product $\lambda_{313}^{\prime \prime} \lambda_{323}^{\prime \prime *}$ depends in a nontrivial way on the spectrum of sparticle masses and mixing angles. As a first step, we derived an upper bound on the $R_{p}$-violating couplings by performing a general scan over the parameter space of the MSSM at the weak scale. According to the standard lore on the hierarchy problem, we varied the soft squark masses $m_{Q_{3}}, m_{U_{3}}$ and $m_{D_{3}}$, the gaugino mass $M_{2}$ and the charged Higgs mass $m_{H^{+}}$between $100 \mathrm{GeV}$ and $1 \mathrm{TeV}$, the squark trilinear couplings $A_{t}, A_{b}$ and the $\mu$ parameter between $-1 \mathrm{TeV}$ and $1 \mathrm{TeV}$, and $\tan \beta$ between 1 and 50 , with the constraint that the mass spectrum of superpartners is not already excluded by direct searches. The result is that, for any choice of the MSSM parameters, the upper limit on the $R_{p}$-violating couplings is of order $\left|\lambda_{313}^{\prime \prime} \lambda_{323}^{\prime \prime *}\right|^{1 / 2}<0.033$ or lower (the weakest bound is obtained when $m_{U_{3}}, m_{D_{3}}$ and $|\mu|$ get their maximal value). This is significantly stronger than the upper limit $\left|\lambda_{313}^{\prime \prime} \lambda_{323}^{\prime \prime *}\right|^{1 / 2}<0.12$ quoted in 11] for the case $m_{\tilde{b}_{R}}=1 \mathrm{TeV}$, $m_{\tilde{t}_{R}}=900 \mathrm{GeV}$. However, the authors of [11] neglected in their analysis the contribution of the diagram with chargino exchange shown in fig. 1]e, as well as the effect of squark mixing and QCD corrections.

\section{Constraints from flavor symmetries}

As noticed above, flavor changing processes allow to set bounds only on products of two different $\lambda_{3 j k}^{\prime \prime}$ couplings. To discuss the implications for single superparticle production at colliders, however, it would be interesting to translate these bounds into bounds on the individual couplings. Two extreme but simple-minded choices that are often made in the literature consist in assuming either degenerate couplings or only one non-vanishing coupling: in the first case, $\lambda_{312}^{\prime \prime}=\lambda_{313}^{\prime \prime}=\lambda_{323}^{\prime \prime} \equiv \lambda^{\prime \prime}$, the limit from $K^{0}-\bar{K}^{0}$ mixing is simply $\lambda^{\prime \prime}<0.033$ or lower, whereas in the second case the only non-vanishing coupling is unconstrained. However, none of these two choices is natural, as can be easily realized considering flavor models based on horizontal symmetries. As a representative case we shall concentrate here on the class of models based on a U(1) flavor symmetry, but similar 
considerations could be made for all other realistic flavor models. In abelian flavor models, the Yukawa couplings for up-type and down-type quarks are of order $Y_{i j}^{U} \sim \epsilon^{h_{2}+q_{i}+u_{j}}$ and $Y_{i j}^{D} \sim \epsilon^{h_{1}+q_{i}+d_{j}}$ respectively, while the R-parity and baryon number violating couplings are of order $\lambda_{i j k}^{\prime \prime} \sim \epsilon^{u_{i}+d_{j}+d_{k}}$, where $\epsilon \sim 0.2$ is a parameter of the order of the Cabibbo angle and $h_{i}, q_{i}, u_{i}$ and $d_{i}$ denote the charges of the corresponding MSSM superfields (in the basis of interaction eigenstates) under the additional $\mathrm{U}(1)$ symmetry. It has been shown [20]-22 that the correct pattern of quark masses and mixing angles can be reproduced only with the following two sets of charge assignments:

$$
\begin{aligned}
& \left(q_{13}, q_{23}\right)=(3,2), \quad\left(u_{13}, u_{23}\right)=(5,2), \quad\left(d_{13}, d_{23}\right)=(1,0), \\
& \left(q_{13}, q_{23}\right)=(-3,2), \quad\left(u_{13}, u_{23}\right)=(11,2), \quad\left(d_{13}, d_{23}\right)=(7,0)
\end{aligned}
$$

where $q_{i j} \equiv q_{i}-q_{j}$ etc. In the basis of interaction eigenstates, the hierarchy among the $\lambda_{3 j k}^{\prime \prime}$ couplings follows the pattern:

$$
\lambda_{312}^{\prime \prime} \approx \lambda_{313}^{\prime \prime} \approx \kappa \lambda_{323}^{\prime \prime}
$$

where $\kappa=\epsilon$ in case I (i.e. a mild hierarchy) and $\kappa=\epsilon^{7}$ in case II (a strong hierarchy). However, the hierarchy is attenuated in the basis of eq. (迎), where the quarks are mass eigenstates. Following Refs. 223, 20, 21] the quark mixing matrices can be evaluated at leading order in $\epsilon$ upon diagonalization of the Yukawa matrices. We found that in the basis of quark mass eigenstates the hierarchy among the $\lambda_{3 j k}^{\prime \prime}$ couplings follows the pattern of eq. (6), with $\kappa=\epsilon$ in case I and $\kappa=\epsilon^{3}$ in case II. In the presence of such hierarchy, the upper limit on the unsuppressed coupling $\lambda_{323}^{\prime \prime}$ coming from $K^{0}-\bar{K}^{0}$ mixing is rescaled by a factor of $\kappa^{-1 / 2}$ with respect to the case of degenerate couplings, while the upper limit on the suppressed couplings $\lambda_{312}^{\prime \prime}$ and $\lambda_{313}^{\prime \prime}$ is rescaled by a factor of $\kappa^{1 / 2}$ : the resulting bounds are $\lambda_{323}^{\prime \prime}<0.07, \lambda_{312}^{\prime \prime} \approx \lambda_{313}^{\prime \prime}<0.015$ in case I and $\lambda_{323}^{\prime \prime}<0.37, \lambda_{312}^{\prime \prime} \approx \lambda_{313}^{\prime \prime}<0.003$ in case II. Thus, even in the less stringent situation, the upper bound on the largest R-parity violating (s)top coupling is at most of order $($ few $) \times 10^{-1}$, i.e. one order of magnitude below the bounds assumed in Refs. [4]- [8].

\section{Implications for single superparticle production}

We are now ready to discuss the implications of our results for single superparticle production at colliders. In [4] the production of single top squarks via $R_{p}$ violation in $p \bar{p}$ collisions at the Tevatron was studied. If some of the couplings $\lambda_{3 j k}^{\prime \prime}$ are greater than $10^{-2}$ or so, the rate for the production of a single light stop $\tilde{t}_{1}$ in $p \bar{p}$ collisions at $\sqrt{S}=2 \mathrm{TeV}$ may exceed the rate for stop-antistop pair production, due to the greater phase space available. Thus, $R_{p}$ violation could be the favorite scenario for the observation of supersymmetric particles at the Tevatron. The authors of [4] considered a supergravity-inspired scenario, where at the Grand Unified Theory (GUT) scale the common gaugino mass is $m_{1 / 2}=150 \mathrm{GeV}$, the scalar trilinear coupling is $A_{0}=-300 \mathrm{GeV}$ and the common scalar mass $m_{0}$ is varied 
in a range between 50 and $500 \mathrm{GeV}$. The ratio of the Higgs vacuum expectation values is chosen to be $\tan \beta=4$ and the Higgs mass parameter $\mu$, whose absolute value is fixed by electroweak symmetry breaking, is chosen to be positive. The three $R_{p}$-violating (s)top couplings were taken to be degenerate, $\lambda_{312}^{\prime \prime}=\lambda_{313}^{\prime \prime}=\lambda_{323}^{\prime \prime} \equiv \lambda^{\prime \prime}$. The signal coming from single stop production in $p \bar{p}$ collisions, followed by the $R_{p}$-conserving decay $\tilde{t}_{1} \rightarrow b+\tilde{\chi}_{1}^{+}$, with $\tilde{\chi}_{1}^{+} \rightarrow l+\nu+\tilde{\chi}_{1}^{0}$, was considered in [4] together with the Standard Model background. The conclusion was that, for $180<m_{\tilde{t}_{1}}<325 \mathrm{GeV}$ and $\lambda^{\prime \prime}>0.02-0.06$, it should be possible to discover the top squark at run II of the Tevatron, otherwise the limit on the $R_{p}$-violating couplings could be lowered to $\lambda^{\prime \prime}<0.01-0.03$ at $95 \%$ confidence level. Moreover, existing data from run I should allow for a reduction of the limit to $\lambda^{\prime \prime}<0.03-0.2$ for $180<m_{\tilde{t}_{1}}<280 \mathrm{GeV}$. We computed the upper bound on $\lambda^{\prime \prime}$ coming from $K^{0}-\bar{K}^{0}$ mixing in the minimal supergravity scenario considered by the authors of [1], for the same choice of parameters. The resulting limits are of order $\lambda^{\prime \prime}<0.015-0.020$ for a light stop mass ranging between 180 and $325 \mathrm{GeV}$, as shown in fig. 2. In the same figure, the limits resulting from the general scan over the MSSM parameters described above are shown, and it can be seen that they never exceed $\lambda^{\prime \prime}<0.033$. The (weaker) limits coming from rare decays of the $B^{+}$boson (see section 1) are also shown. In summary, under the assumption of degenerate $R_{p}$-violating (s)top couplings, the discovery of single stop production via R-parity violation at the Tevatron turns out to be unlikely.

In the presence of a hierarchy among the couplings such as that of eq. (6), suggested by $\mathrm{U}(1)$ flavor models, the lower limit for single stop discovery on the unsuppressed coupling $\lambda_{323}^{\prime \prime}$ must be rescaled by a factor of $\left(0.95 \kappa^{2}+0.05\right)^{-1 / 2}$ with respect to the case of degenerate couplings, taking into account that the relative contribution of $\lambda_{323}^{\prime \prime}$ to the total cross section $p \bar{p} \rightarrow \tilde{t}_{1}$ is roughly $5 \%$. On the other side, as discussed in section 3 , the upper limit on $\lambda_{323}^{\prime \prime}$ coming from $K^{0}-\bar{K}^{0}$ mixing is rescaled by a factor of $\kappa^{-1 / 2}$. In case I $(\kappa=\epsilon)$ the upper limit from $K^{0}-\bar{K}^{0}$ mixing becomes $\lambda_{323}^{\prime \prime}<0.034-0.045$, while the lower limit for single stop discovery becomes $\lambda_{323}^{\prime \prime}>0.07-0.20$ : no room for single stop discovery at the Tevatron is left. In case II $\left(\kappa=\epsilon^{3}\right)$ the upper limit from $K^{0}-\bar{K}^{0}$ mixing becomes $\lambda_{323}^{\prime \prime}<0.17-0.22$, while the lower limit for single stop discovery becomes $\lambda_{323}^{\prime \prime}>0.09-0.27$. Although single stop discovery at the Tevatron cannot be excluded in this case, most of the parameter space considered in [4] is ruled out.

A very similar discussion can be made concerning the results of [7], where the production of single gluinos at hadron colliders, $p \bar{p}(p p) \rightarrow \bar{t} \tilde{g}(t \tilde{g})$, was studied. Such process occurs through the exchange of a virtual squark, produced in the R-parity violating interaction between two quarks. The authors of [7] considered a supergravity-inspired scenario where at the GUT scale $m_{0}=1 \mathrm{TeV}, A_{0}=-1 \mathrm{TeV}, \tan \beta=10, \mu>0$ and $m_{1 / 2}$ is varied between 120 and $400 \mathrm{GeV}$, i.e. a region of the parameter space where the squarks are heavier than the gluino (with the possible exception of the light stop). The $R_{p}$-violating stop couplings were taken to be degenerate, $\lambda_{3 j k}^{\prime \prime} \equiv \lambda^{\prime \prime}$. The conclusion was that, if the gluino is lighter than $400 \mathrm{GeV}$ and $\lambda^{\prime \prime}$ is of order 1 , it will be possible to detect single gluino production at the Run II of the Tevatron. At the LHC the process can potentially 
be seen with the gluino lighter than $1 \mathrm{TeV}$ and $\lambda^{\prime \prime}$ of order 0.01 . We computed the upper bound on $\lambda^{\prime \prime}$ coming from $K^{0}-\bar{K}^{0}$ mixing, with the choice of parameters considered in [7]. The result is that $\lambda^{\prime \prime}<0.028-0.035$ for a gluino mass ranging between $350 \mathrm{GeV}$ and $1 \mathrm{TeV}$. Thus, under the assumption of degenerate $\lambda_{3 j k}^{\prime \prime}$ couplings, the discovery of single gluino production at the Tevatron is unlikely, while room is left for discovering the same process at the LHC. The same conclusion holds in the presence of a moderate hierarchy among the couplings, such as the one suggested by the U(1) flavor models of section 3 .

In 8 the decay $t \rightarrow \bar{b} \bar{d} \tilde{\chi}_{1}^{0}$ was studied. If the light sbottom has a non-negligible righthanded component, the top quark may undergo the $R_{p}$-violating decay $t \rightarrow \bar{b}_{1} \bar{d}$ through the coupling $\lambda_{313}^{\prime \prime}$, followed by the $R_{p}$-conserving decay $\overline{\tilde{b}}_{1} \rightarrow \bar{b} \tilde{\chi}_{1}^{0}$. The authors of [8] considered a scenario where at the weak scale $\mu=-200 \mathrm{GeV}, \tan \beta=1$ and $M_{2}=100$ $\mathrm{GeV}$. With this choice of the parameters the masses of the lightest chargino and neutralino are $m_{\tilde{\chi}_{1}^{+}}=120 \mathrm{GeV}$ and $m_{\tilde{\chi}_{1}^{0}}=55 \mathrm{GeV}$, respectively. The conclusion of [8] was that, if the sbottom $\tilde{b}_{1}$ is lighter than $160 \mathrm{GeV}$ and $\lambda_{313}^{\prime \prime}>0.5$, it will be possible to observe the $R_{p}$-violating top decay at the Run II of the Tevatron, while the observation at the LHC will be possible for $\lambda_{313}^{\prime \prime}>0.1$. The same conclusion holds for the decay $t \rightarrow \bar{b} \bar{s} \tilde{\chi}_{1}^{0}$, driven by the $R_{p}$-violating coupling $\lambda_{323}^{\prime \prime}$. We computed the upper limits on the combination $\left|\lambda_{313}^{\prime \prime} \lambda_{323}^{\prime \prime *}\right|^{1 / 2}$, using for $M_{2}, \mu$ and $\tan \beta$ the same values as in [8], and varying the other MSSM parameters in the way described in section 2. The results are shown in fig. 3 as a function of the right sbottom mass $m_{\tilde{b}_{R}}$ (with our choice of $\mu$ and $\tan \beta$ the mixing in the sbottom sector is negligible). It can be noticed that, when the right sbottom is lighter than $160 \mathrm{GeV}$, the upper bounds on the $R_{p}$-violating couplings are of order $\left|\lambda_{313}^{\prime \prime} \lambda_{323}^{\prime \prime *}\right|^{1 / 2}<0.013$ or lower. Thus we conclude that, if the couplings $\lambda_{313}^{\prime \prime}$ and $\lambda_{323}^{\prime \prime}$ are degenerate (or if there is a hierarchy between the couplings such as the one suggested by $\mathrm{U}(1)$ flavor models), the discovery of $R_{p}$-violating top decays is unlikely. In any case, we cannot expect to observe both the decays $t \rightarrow \bar{b} \bar{d} \tilde{\chi}_{1}^{0}$ and $t \rightarrow \bar{b} \bar{s} \tilde{\chi}_{1}^{0}$.

\section{Conclusions}

In summary, we have improved the existing bounds on the $R_{p}$-violating couplings $\lambda_{3 j k}^{\prime \prime}$, showing that they are typically more stringent than those assumed in [4]- [8]. For the bounds coming from $K^{0}-\bar{K}^{0}$ mixing we have included QCD corrections and a class of diagrams that were neglected in earlier analyses [10]-12]: both effects give sizeable contributions. We have discussed a way of translating the resulting bounds into constraints on the individual couplings, making use of the simplest class of flavor models based on $\mathrm{U}(1)$ horizontal symmetries. Finally, we have shown that our bounds put severe constraints on the possibility of observing single superparticle production via R-parity violation at the Tevatron.

\footnotetext{
${ }^{1}$ Our normalization of the couplings $\lambda_{i j k}^{\prime \prime}$ (eq. 1) differs by a factor of 2 from the one used in [8].
} 


\section{Acknowledgements}

The author thanks Fabio Zwirner for many useful discussions, and Guido Martinelli for providing the revised version of the 'magic numbers' of [18] before publication.

\section{Appendix}

We have calculated the contributions to $K^{0}-\bar{K}^{0}$ mixing coming from the full set of diagrams with $R_{p}$-violating (s)top couplings $\lambda_{3 j k}^{\prime \prime}$. In particular, the contribution of the diagram in fig. [le, which was neglected in earlier analyses [10]-[12], turns out to be sizeable. The calculation has been performed in the basis where the quark masses are diagonal, and all the flavor changing squark mass insertions have been neglected. We have also checked that in the scenarios considered in [4]-[8] the contributions coming from the R-parity conserving sector of the MSSM (i.e. from diagrams with quarks and charged Higgs or squarks and charginos in the internal lines) are negligible. The coefficients $C_{i}$ that appear in eq. (3) are:

$$
\begin{aligned}
& C_{1}= \sum_{i, j=1}^{3} \frac{g^{4}}{128 \pi^{2}} K_{i 1}^{*} K_{i 2} K_{j 1}^{*} K_{j 2} m_{u_{i}}^{2} m_{u_{j}}^{2}\left[I_{0}+2 I_{2} / m_{W}^{2}+I_{4} / 4 m_{W}^{4}\right]\left(m_{u_{i}}^{2}, m_{u_{j}}^{2}, m_{W}^{2}, m_{W}^{2}\right) \\
& \tilde{C}_{1}= \sum_{i, j=1}^{2} \frac{1}{4 \pi^{2}}\left|\lambda_{313}^{\prime \prime} \lambda_{323}^{\prime \prime *}\right|^{2}\left[\left(O_{i 2}^{t} O_{j 2}^{t}\right)^{2} I_{4}\left(m_{b}^{2}, m_{b}^{2}, m_{\tilde{t}_{i}}^{2}, m_{\tilde{t}_{j}}^{2}\right)+\left(O_{i 2}^{b} O_{j 2}^{b}\right)^{2} I_{4}\left(m_{\tilde{b}_{i}}^{2}, m_{\tilde{b}_{j}}^{2}, m_{t}^{2}, m_{t}^{2}\right)\right] \\
& C_{5}=\sum_{i=1}^{2} \frac{g^{2}}{4 \pi^{2}} \lambda_{313}^{\prime \prime} \lambda_{323}^{\prime \prime *}\left(O_{i 2}^{b}\right)^{2} K_{31}^{*} K_{32} m_{t}^{2}\left[I_{2}\left(m_{\tilde{b}_{i}}^{2}, m_{W}^{2}, m_{t}^{2}, m_{t}^{2}\right)+\right. \\
&+\sum_{i, j, k=1}^{2} \frac{1}{4 m_{W}^{2}} I_{4}\left(m_{\tilde{b}_{i}}^{2}, m_{W}^{2}, m_{t}^{2}, m_{t}^{2}\right)+\frac{1}{4 \pi^{2}} \lambda_{313}^{\prime \prime} \lambda_{323}^{\prime \prime} O_{j 2}^{t} O_{k 2}^{t} K_{31}^{*} K_{32}\left[V_{i 1}^{*} O_{j 1}^{t}-\frac{m_{t}}{\sqrt{2} m_{W} \sin \beta} I_{4}\left(m_{\tilde{b}_{i}}^{2}, m_{H^{+}}^{2}, m_{t}^{2}, m_{t}^{2}\right)\right] \times \\
& {\left[V_{i 1}^{t} O_{k 1}^{t}-\frac{m_{t}}{\sqrt{2} m_{W} \sin \beta} V_{i 2} O_{k 2}^{t}\right] I_{4}\left(m_{b}^{2}, m_{\tilde{\chi}_{i}^{+}}^{2}, m_{\tilde{t}_{j}}^{2}, m_{\tilde{t}_{k}}^{2}\right) }
\end{aligned}
$$

and $C_{4}=-C_{5}$. In the above equations, $K_{i j}$ are the CKM matrix elements, $O_{i j}^{t}$ and $O_{i j}^{b}$ are the left-right mixing matrices of the stop and sbottom sectors, and $V_{i j}$ is the mixing matrix of positive charginos as defined in [24]. The functions $I_{n}$ result from integration over the Euclidean momentum $\bar{k}$ of the four particles circulating in the loop:

$$
I_{n}\left(m_{1}^{2}, m_{2}^{2}, m_{3}^{2}, m_{4}^{2}\right)=\int_{0}^{\infty} \frac{\bar{k}^{n} d \bar{k}^{2}}{\left(\bar{k}^{2}+m_{1}^{2}\right)\left(\bar{k}^{2}+m_{2}^{2}\right)\left(\bar{k}^{2}+m_{3}^{2}\right)\left(\bar{k}^{2}+m_{4}^{2}\right)}
$$

In the numerical study of the minimal supergravity scenario, the masses of the supersymmetric particles and the mixing angles at the weak scale have been calculated with ISAJET 
[25], and the common scale $M_{S}$ has been chosen as the geometrical mean of squark and chargino masses.

\section{References}

[1] G. R. Farrar and P. Fayet, Phys. Lett. B 76 (1978) 575.

[2] H. Dreiner, in Perspectives on Supersymmetry, ed. by G. L. Kane, World Scientific, Singapore (1998), p. 462-479, preprint hep-ph/9707435.

[3] F. Zwirner, Phys. Lett. B 132 (1983) 103.

[4] E. L. Berger, B. W. Harris and Z. Sullivan, Phys. Rev. Lett. 83 (1999) 4472.

[5] B. Allanach et al., to appear in the Proceedings of the Workshop on Physics at Run II - Supersymmetry/Higgs, Fermilab (1998), preprint hep-ph/9906224.

[6] T. Plehn, Phys. Lett. B 488 (2000) 359.

[7] M. Chaichian, K. Huitu and Z. H. Yu, Phys. Lett. B 490 (2000) 87.

[8] K. J. Abraham et al., preprint hep-ph/0007280.

[9] J. L. Goity and M. Sher, Phys. Lett. B 346 (1995) 69; erratum ibid. B 385 (1996) 500.

[10] R. Barbieri and A. Masiero, Nucl. Phys. B 267 (1986) 679.

[11] C. E. Carlson, P. Roy and M. Sher, Phys. Lett. B 357 (1995) 99.

[12] B. de Carlos and P. L. White, Phys. Rev. D 55 (1997) 4222.

[13] G. Bhattacharyya, D. Choudhury and K. Sridhar, Phys. Lett. B 355 (1995) 193.

[14] J. M. Yang, preprint hep-ph/9905486.

[15] O. Lebedev, W. Loinaz and T. Takeuchi, Phys. Rev. D 62 (2000) 015003.

[16] D. Chang and W. Y. Keung, Phys. Lett. B 389 (1996) 294.

[17] D. E. Groom et al. (Particle Data Group), Eur. Phys. Jour. C 15 (2000) 1.

[18] M. Ciuchini et al., JHEP 10 (1998) 008, preprint hep-ph/9808328 v2.

[19] S. Herrlich and U. Nierste, Nucl. Phys. B 419 (1994) 292, Phys. Rev. D 52 (1995) 6505, Nucl. Phys. B 476 (1996) 27.

[20] E. Dudas, S. Pokorski and C. A. Savoy, Phys. Lett. B 356 (1995) 45. 
[21] P. Binétruy, S. Lavignac and P. Ramond, Nucl. Phys. B 477 (1996) 353.

[22] E. J. Chun and A. Lukas, Phys. Lett. B387 (1996) 99; K. Choi, E. J. Chun and H. Kim, Phys. Lett. B 394 (1997) 89; K. Choi, E. J. Chun and K. Hwang, Phys. Rev. D 60 (1999) 031301.

[23] L. J. Hall and A. Rasin, Phys. Lett. B 315 (1993) 164; M. Leurer, Y. Nir and N. Seiberg, Nucl. Phys. B 420 (1994) 468.

[24] H. E. Haber and G. L. Kane, Phys. Rept. 117 (1985) 75.

[25] H. Baer et al., in Proceedings of the Workshop on Physics at Current Accelerators and Supercolliders, ed. by J. Hewett et al., Argonne National Laboratory (1993), preprint hep-ph/9804321. 


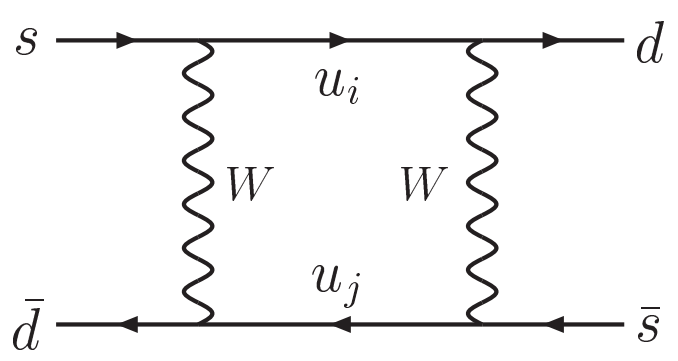

(a)

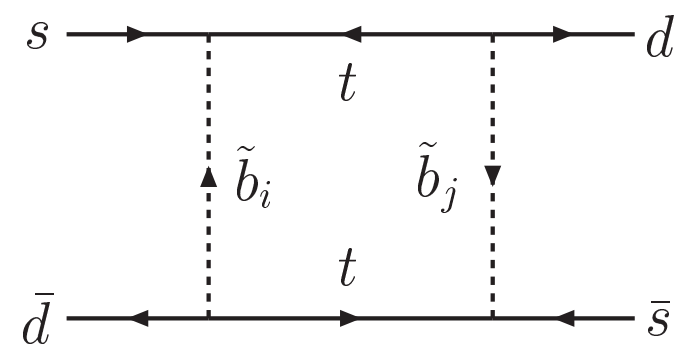

(b)

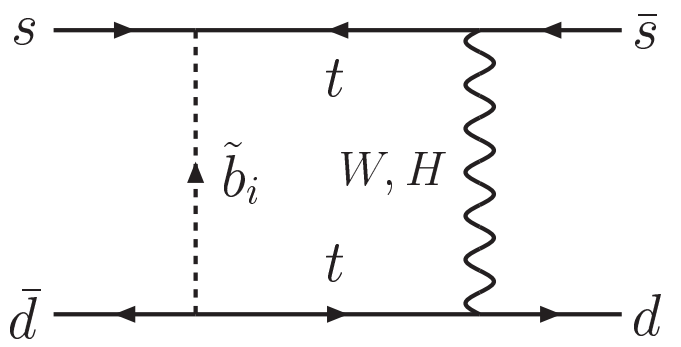

(d)

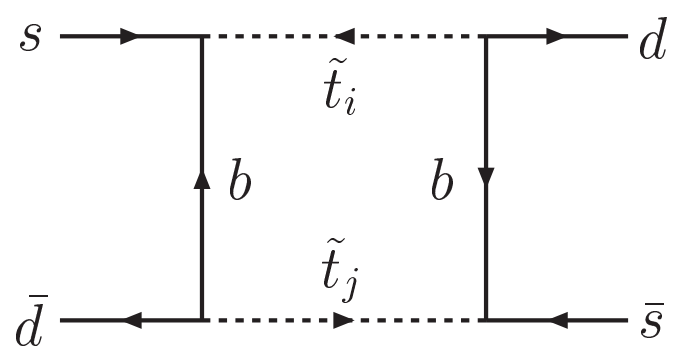

(c)

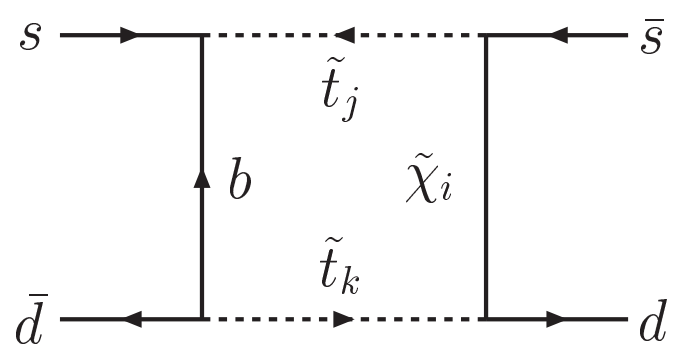

(e)

Figure 1: Standard Model diagram (a) and diagrams with $R_{p}$-violating couplings (b-e) that give the dominant contributions to $K^{0}-\bar{K}^{0}$ mixing. The arrows indicate the flow of baryon number. 


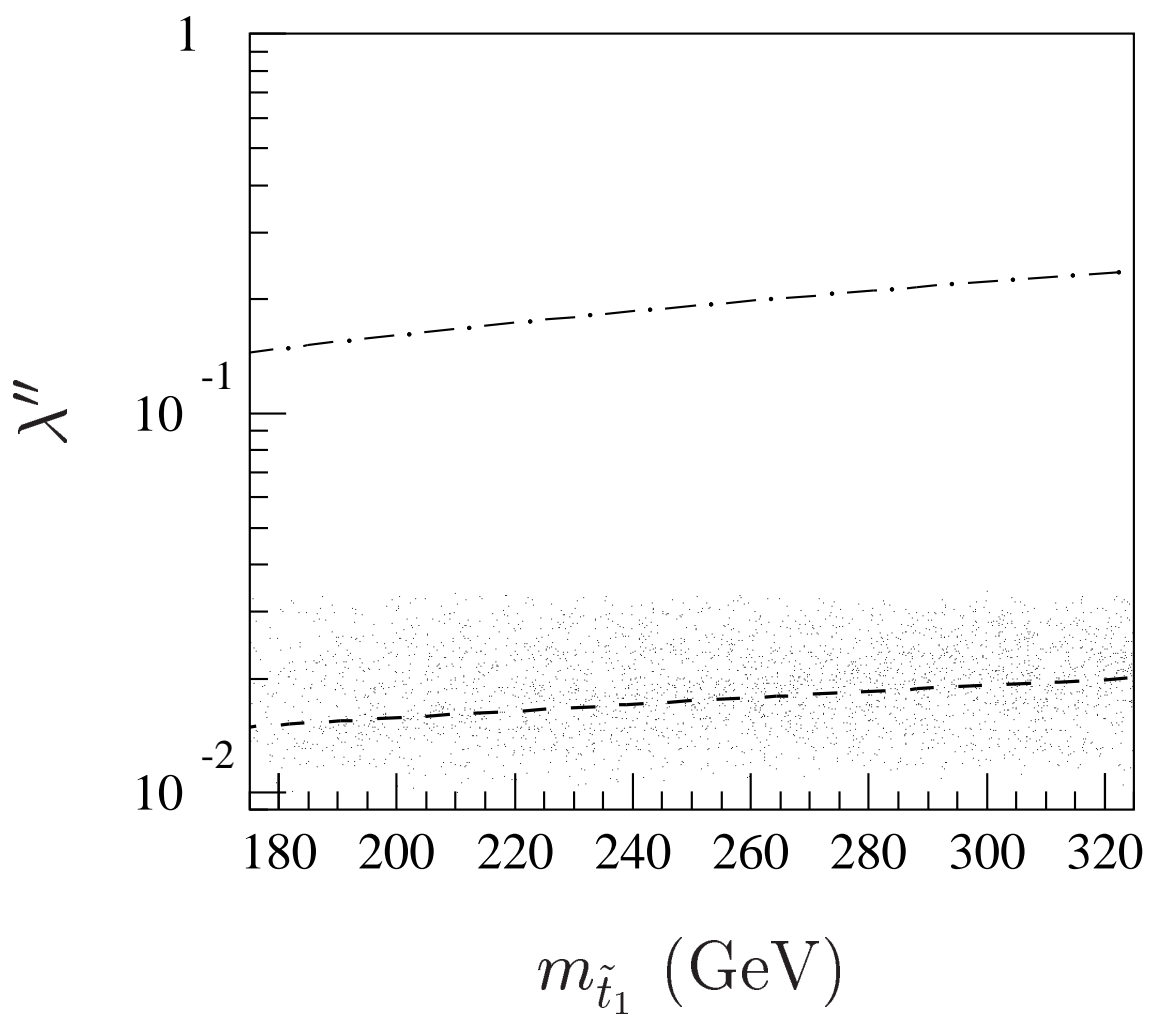

Figure 2: Upper limits on $\lambda^{\prime \prime}$ coming from $K^{0}-\bar{K}^{0}$ mixing (dashed line) and $B$ rare decays (dot-dashed line) in the scenario relevant to single stop production, with the choice of parameters considered in [4]. The scattered points are the limits from $K^{0}-\bar{K}^{0}$ mixing that result from a wide scan over the MSSM parameter space. 


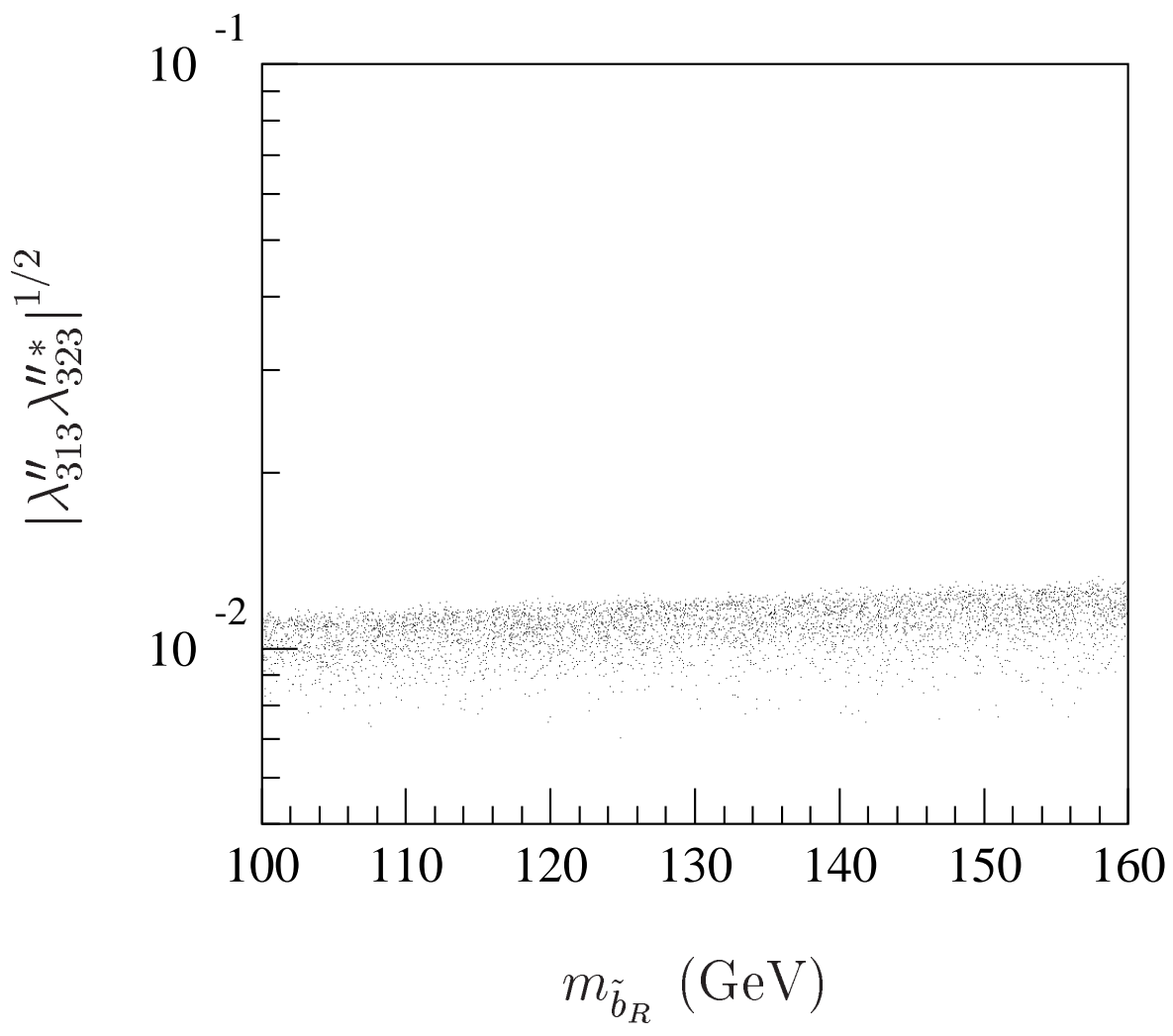

Figure 3: Upper limits on $\left|\lambda_{313}^{\prime \prime} \lambda_{323}^{\prime \prime *}\right|^{1 / 2}$ coming from $K^{0}-\bar{K}^{0}$ mixing, in the scenario relevant to $R_{p}$-violating top decays [8]. The scattered points are the limits that result when $M_{2}=$ $100 \mathrm{GeV}, \tan \beta=1, \mu=-200 \mathrm{GeV}$ and the other MSSM parameters are varied as described in the text. 